\title{
Disentangling dynamic affect trajectories for distinct depression courses during the COVID-19 pandemic
}

\author{
Gabriela Lunansky ${ }^{1, *}$, Ria HA Hoekstra ${ }^{1, *}$, Tessa F Blanken ${ }^{1}$ \\ 1 Department of Psychological Methods, University of Amsterdam, 1018 WT Amsterdam \\ * Shared first-authors
}

\section{Corresponding author}

Correspondence concerning this article should be addressed to Tessa Blanken, Department of Psychological Methods, University of Amsterdam, Nieuwe Achtergracht 129-B, 1018 WS Amsterdam. E-mail: t.f.blanken@uva.nl

\section{Declaration of interest}

None.

\section{Funding Statement}

GL is supported by ERC Consolidator Grant no. 647209, awarded to Denny Borsboom.

RHA is supported by NWO Research Talent Grant no. 406-18-532 awarded to RHA Hoekstra. TFB is supported by BIAL Foundation Grant no. 284/20 awarded to TF Blanken.

\section{Acknowledgements}

We would like to thank Tony Cunningham and colleagues from Boston College for collecting and sharing this unprecedented dataset. We would like to thank Denny Borsboom and Jonas Haslbeck for their very helpful comments on earlier drafts of this manuscript. 


\section{Abstract}

Background. Why does adversity lead to mental health complaints in some, but not others? Individual differences in the development of depressive complaints are related to the regulation of affect states. The COVID-19 pandemic has caused a prolonged period of perturbations to the daily lives of people across the globe, providing an unparalleled opportunity to investigate how fluctuations in positive and negative affect relate to the evolution of mood complaints.

Methods. 228 participants from the Boston College daily sleep and well-being survey completed at least 20 assessments of positive and negative affect and depression complaints between March $20^{\text {th }} 2020$ and June $26^{\text {th }} 2020$. We explored affect trajectories throughout this period and estimated longitudinal multilevel network models. Furthermore, we investigated how individual network structures relate to changes in depression severity over time.

Results. On average, positive affect was reported somewhat higher than negative affect. However, when separating affect trajectories based on the individuals' depressive complaints, we identified that individuals consistently experiencing depressive complaints report higher levels of negative affect compared with positive affect. Contrary, individuals consistently reporting no depressive complaints show opposite results. Furthermore, we found many and strong associations in the multilevel network between the distinct affect states and depressive complaints. Lastly, we established that the higher the connectivity of an individual's network, the larger their change in depressive complaints is.

Conclusions. We conclude that affect fluctuations are directly related to the development of depressive complaints, both within- and across individuals, and both within a single measurement moment and over time. 


\section{Introduction}

The COVID-19 pandemic has impacted lives and societies across the globe in an unprecedented way, imposing a prolonged period of stress on people worldwide (Brown et al., 2020; Moore \& Kolencik, 2020). Prolonged stress is among the most robust risk factors for developing mental health problems in general and mood disorders such as depression (e.g., Bale, 2006; Calabrese et al., 2009; Guille et al., 2014; Hölzel et al., 2011; Kalisch et al., 2017; McEwen, 2000). Understanding why some people develop mental health complaints in the face of adversity while others do not, remains one of the most pressing questions in clinical psychology today. The current COVID-19 crisis provides an unparalleled opportunity to investigate how pronounced changes in daily lives might result in the development of mental health complaints. Interestingly, the effect of COVID-19 regulations on mental health seems to be multifaceted; while mentalhealth problems were initiated or worsened in some, in others, their problems alleviated (e.g., Kocevska et al., 2020). The COVID-19 pandemic can thus be viewed as imposing prolonged perturbations on people's daily lives from which they may experience fluctuations in their mental health.

Many studies have found that individual differences in the development of depressive complaints are associated with the regulation of affect states (e.g., Dejonckheere et al., 2019; Garnefski \& Kraaij, 2006; Gross, 1999; Joormann \& Gotlib, 2010; Joormann \& Stanton, 2016). Affect states refer to various feeling states, such as emotions and moods (Abrams et al., 2013). Typically, affect states are divided into positive affect (PA), for example, feeling inspired or enthusiastic, and negative affect (NA), such as feeling afraid or upset (Watson et al., 1988). PA is generally associated with a reduced risk of developing mood symptoms (Khazanov \& Ruscio, 2016; Wichers et al., 2010), while NA is related to an increased risk of developing depressive symptoms (Wichers et al., 2007). Researchers collect multiple assessments of mood complaints and affect states to investigate potential individual differences in the development of mental disorders (e.g., see Kramer et al., 2014; Kuranova et al., 2020; Wichers et al., 2015). However, longitudinal studies focusing on the relation between mood and affect often take place within a short time period, such as two weeks (e.g., see Schoevers et al., 2020). Since symptoms mostly evolve over a longer period, it is possible that brief studies do not fully capture potentially meaningful changes in the development of symptomatology. In addition, participants may not experience enough perturbations or disturbances in their daily lives during these brief time periods, which hampers the investigation of the relation between fluctuations in affect and the development of mood complaints. The COVID-19 pandemic has created an exceptional situation of prolonged perturbations to investigate how individual affect trajectories are related to the dynamic course of depressive complaints (Han et al., 2021).

The current paper uses data that are part of a longitudinal investigation from the Boston College, in which the repercussions of the COVID-19 pandemic on mental health are investigated (Cunningham et al., 2021). The studied period (March $20^{\text {th }} 2020$ until June $26^{\text {th }} 2020$ ) commenced a day after the first "stay-at-home" order was issued in California, which covers the moments leading up to the first large COVID-19 wave in the US. Clearly, this was a period of great uncertainty, and many effects on mental health problems during this time have been reported across all levels of society (Grolli et al., 2021; Kaufman et al., 2020; Pfefferbaum \& North, 2020; Van Lancker \& Parolin, 2020). We investigate how fluctuations in positive and negative affect relate to the evolution of mood complaints in the face of these perturbations. We study the affect trajectories of all individuals, and examine whether these trajectories differ for individuals with different depressive courses. In addition, we study which potential mechanisms may underlie the individual 
trajectories by estimating longitudinal multilevel network models (Borsboom \& Cramer, 2013; Epskamp, van Borkulo, et al., 2018a; Epskamp, Waldorp, et al., 2018). These network models can estimate the direct interactions between the affect states and the depressive complaints for every individual and across individuals, within one measurement moment and across all measurement moments.

\section{Methods}

The preregistration of this study can be found at the online repository of the Open Science Framework (https://osf.io/fw3np).

\section{Participants}

The data were obtained through the Boston College daily sleep and well-being survey (Cunningham et al., 2021). The study was set-up during the first wave of COVID-19 (March $20^{\text {th }} 2020$ until August $5^{\text {th }}$ 2020) and participants were recruited online. All English-speaking individuals older than 18 were eligible to participate in the study, resulting in $\mathrm{N}=1,518$ enrolled participants (mean $\pm \mathrm{SD}$ age $35.2 \pm 15.1$ years old, range 18-90 years old). The participants provided informed consent, and the study received ethical approval from the Institutional Review Board at Boston College. More details on the study and recruitment can be found in Cunningham et al., 2021.

\section{Procedure}

The study started with a demographic survey, and upon completion participants received daily surveys on their sleep and well-being. The daily surveys were divided into a short and a full version, where the full version included additional questions containing validated assessments of mood (Positive and Negative Affect Schedule [PANAS]; Watson et al., 1988) and depression complaints (Patient Health Questionnaire9 [PHQ-9]; Kroenke et al., 2001). The full version was sent on the first three days of the study enrollment. After enrollment, the long survey was sent on two randomly selected days of the week, and the short version was sent on the five remaining days. In addition to the daily surveys, three rounds of assessments included validated measures on mental health, personality, emotion regulation, and events surrounding COVID-19. These assessments were launched on May 19 2020, June 16 2020, and June 29 2020, respectively. For a more detailed description on the assessments, we refer to Cunningham et al., 2021.

\section{Materials}

For the current study we include the demographics survey, PANAS items, and PHQ-9 assessments from the full version of the questionnaire.

PANAS. The PANAS is a 20-item questionnaire on the experience of positive (e.g., enthusiastic) and negative (e.g., scared) affects rated on a five-point Likert scale ranging from 1 ("very slightly/ not at all") to 5 ("extremely") (Watson et al., 1988). To reduce the number of variables for better power of the conducted statistical analyses (i.e., the estimated network models described in the "Statistical Analyses" section of the current paper), we selected the ten items of the PANAS that have been validated in the shortform (Mackinnon et al., 1999). For the assessment of PA, these include: inspired, alert, excited, enthusiastic, determined, and for NA, these include: afraid, upset, nervous, scared, distressed. In the survey participants were explicitly asked to rate how they felt in the current moment ("For each of the following attributes, indicate which description best describes how you currently feel, right now in the moment"). 
PHQ-9. The PHQ-9 is a 9-item questionnaire to measure depression severity by assessing each of the 9 DSM-IV criteria for depression on a four-point Likert scale ranging from 0 ("not at all") to 3 ("nearly every day") (Kroenke et al., 2001). In the current implementation, the item on suicidal thoughts was omitted. Participants were asked to rate the severity of complaints over the last several days ("In the last several days, how often have you been bothered by any of the following problems: not at all, some of the time, more than half of the time, almost all of the time").

\section{Data selection and pre-processing}

Study period. Since the full version of the questionnaire was sent out twice a week on random occasions, we selected the study period from March $20^{\text {th }} 2020$ until June $26^{\text {th }} 2020$, ending three days after the final full survey was sent out. Of the total number of participants enrolled in this study, $N=1,355$ completed assessments between the selected study period.

Pre-processing of assessments. Sometimes participants completed the full survey multiple times on a single day: on 79 occasions, the survey was completed twice, and on two occasions the survey was completed three times. For these 81 assessments we choose the survey that was completed first. Inspecting the response rate of the full survey over the study period, a clear three-day interval pattern is seen (see Supplement A, Figure 1-2). Therefore, we choose to group the days into 'measurement occasions', defined by a three-day window (e.g., March $20^{\text {th }}-22^{\text {nd }} 2020$ is measurement occasion 1). In this way, the entire study period is grouped into 33 measurement occasions of three days each. The advantage of this grouping is twofold. First, it circumvents the problem of large differences in the number of completed surveys per assessment. Second, given that the full surveys were sent out randomly twice a week, dividing the assessments into three-day intervals makes the time between two completed surveys more consistent. In case participants completed multiple surveys within one measurement occasion, we averaged their responses. Following the recommendations for estimating a multilevel model (see statistical analyses below), we selected participants who completed surveys for at least twenty measurement occasions, resulting in a final sample size of $\mathrm{N}=228$ participants (out of 1355). Thus, for each included participant we have completed data for a minimum of 20 and maximum of 33 measurement occasions.

\section{Statistical analyses}

First, we inspect the affect trajectories for positive and negative affect states over the studied time period during the COVID-19 pandemic. We plot the smoothed means of each affect state over time using locally estimated scatterplot smoothing (i.e., a loess curve). Loess regression is a non-parametric method that fits least squares regressions in localized subsets of the data (Cleveland, 1979). The amount of smoothing that is applied depends on the number of data points that are used in each local regression (i.e., the neighborhood), and is controlled by setting the smoothing parameter $\alpha$ between 0 and 1 . The larger the values for $\alpha$, the more data points are being selected in the neighborhood (i.e., $n \alpha$ data points are selected, where $n$ represents the total number of datapoints). More datapoints in the local regression results in smoother functions, that are more robust to fluctuations in the data. We set the smoothing parameter to $\alpha=0.2$ in order to aid the visualization of patterns in the data, without losing sensitivity of fluctuations in the data. 
Second, we aim to investigate whether potential differences in affect trajectories exist depending on the course of depressive complaints. To investigate this, we define subgroups based on clinically meaningful in- or decrease of depressive complaints, defined as a 5-point difference in their PHQ-9 total score (Lowe et al., 2004; Round et al., 2020). In addition, we will investigate whether we can differentiate between participants who consistently do not experience depressive complaints, defined as a PHQ-9 score of four or lower on all assessments; participants who experience occasional depressive complaints, defined as a PHQ-9 score higher than four on at least one assessment (and lower than four on at least one other assessment); and participants who experience consistent depressive complaints, defined as a PHQ-9 score consistently higher than four on all assessments (Kroenke et al., 2001).

Third, while these explorations will shed light on the relation between affect trajectories and depression course, they do not model the interactions among affect states and depressive complaints directly. Therefore, we will more directly investigate their interplay by estimating a multi-level network model including both affect states and depressive complaints. To estimate the relations while taking the longitudinal structure of the data into account we estimate a two-step multi-level GVAR model as implemented in the mlVAR package (Epskamp et al., 2017). Estimating a multi-level GVAR has two major benefits: (1) a single model can be estimated, leading to an adequately powered analysis, and (2) the withinperson effects (reflecting intra-individual differences) can be separated from between-person effects (reflecting inter-individual differences) (Epskamp, van Borkulo, et al., 2018b).

The multi-level GVAR network model consists of nodes, which represent variables (in the current study: affect states and depressive complaints), and edges, which represent the direct conditional associations between the nodes (Borsboom, 2017; Cramer et al., 2016; Epskamp, Borsboom, et al., 2018). The edges in the multi-level GVAR network are computed from partial correlations, meaning they portray the unique association among two variables after controlling for all other variables in the network. Edges can be positive or negative, indicating the corresponding nature of the associations between the nodes (Epskamp, Borsboom, et al., 2018).

The mlVAR package estimates three network structures: (a) a temporal network, (b) a contemporaneous network, and (c) a between-persons network (Epskamp, van Borkulo, et al., 2018b). The temporal network indicates how well a variable predicts another variable at the next time point, while controlling for all variables at the current time point. For example, a direct association from the depression complaint 'trouble concentrating' to the NA 'feeling distressed' indicates that having 'trouble concentrating' now predicts 'feeling distressed' at the next time point, taking into account all other current affect states and depressive complaints. In the contemporaneous network we control for all these temporal effects, and show the unique association among variables within the same time window. For example, a direct and positive edge between 'feeling distressed' and 'feeling afraid' indicates that, within the same time window, these two negative affect states are positively associated, after removing the lagged effects. Finally, the between-persons network shows the relationships among the means of persons in the data. For example, a positive edge between 'trouble concentrating' and 'feeling distressed' would indicate that persons who have, on average, more trouble concentrating also, on average, feel more distressed.

Fourth, based on the theorized role of affect dynamics in the course of depressive complaints, we expected the network structure to differ between people who experienced a meaningful change in their depressive 
complaints (either aggravation or alleviation) and people who did not experience such a meaningful change. Specifically, we expect that in more strongly connected networks, change in one affect state or complaint can more easily "cascade" into other affect states and complaints, thereby resulting in higher overall changes in depressive complaints over time. To explore this hypothesis we computed, for each participant, the average absolute strength of their temporal associations (i.e., their density; Oreel et al., 2019). Subsequently, we correlated their density to the maximum change in their PHQ-9 score over time. Note that this change can be either positive, indicating an aggravation of their depressive complaints, or negative, indicating an alleviation of their depressive complaints.

All analyses were performed in R (version 4.0.5) using the packages 'ggplot2', 'mlVAR', 'qgraph', 'dplyr', 'nnet', and 'ggpubr'. The derived data used for analyses and corresponding code can be found on the Open Science Framework (OSF): https://osf.io/2zh4f/.

\section{Results}

\section{Sample characterization}

We included 228 participants that completed assessments on at least 20 measurement occasions within March $20^{\text {th }} 2020$ and June $26^{\text {th }} 2020$. The majority of the participants was female $(n=186,81.58 \%)$ and on average, (mean $\pm \mathrm{SD}) 45 \pm 19$ years.

First, Figure 1(a) shows the affect trajectories across the 33 measurement occasions for all participants concertedly. Averaging over all participants shows that PA is generally rated somewhat higher than NA, and that this is consistent over the entire study period.

Second, we inspected the affect trajectories for different groups based on (i) their change in depressive complaints over time, and (ii) the severity of the experienced depressive complaints. Over the studied time period, 116 participants experienced a meaningful change in their depression symptomatology: 56/116 participants (48.3\%) experienced an aggravation in their depressive complaints (i.e., an increase in PHQ-9 score of at least 5), and for 60/116 participants (51.7\%) their depressive complaints alleviated (i.e., a decrease in PHQ-9 score of at least 5). Splitting the affect trajectories for each of these groups, shown in figure 1(b), indicates that in both groups, on average, the affect states are more intertwined. Interestingly, no marked differences are seen in participants who experienced an aggravation of their complaints compared with participants who experienced an alleviation of their complaints.

Grouping participants into the consistency of their depressive complaints, we found that 50/228 participants (21.9\%) were consistently without depressive complaints, 137/228 participants (60.1\%) experienced depressive complaints at least occasionally, and 41/228 participants (18.0\%) experienced depressive complaints consistently. Figure 1(c) shows the affect trajectories for each of these groups. Here, we see clear differences in affect trajectories across the three groups: in people without depressive complaints there is, on average, a clear distinction between the PA scores, which are rated relatively high, and the NA scores, which are all rated consistently low. In the people with occasional depressive complaints, the PA scores are, on average, still rated higher than the NA scores, but the distinction is less clear. Finally, in people who consistently experience depressive complaints, the ratings of the positive and NA states have flipped, as NA is, on average, rated higher than PA. 

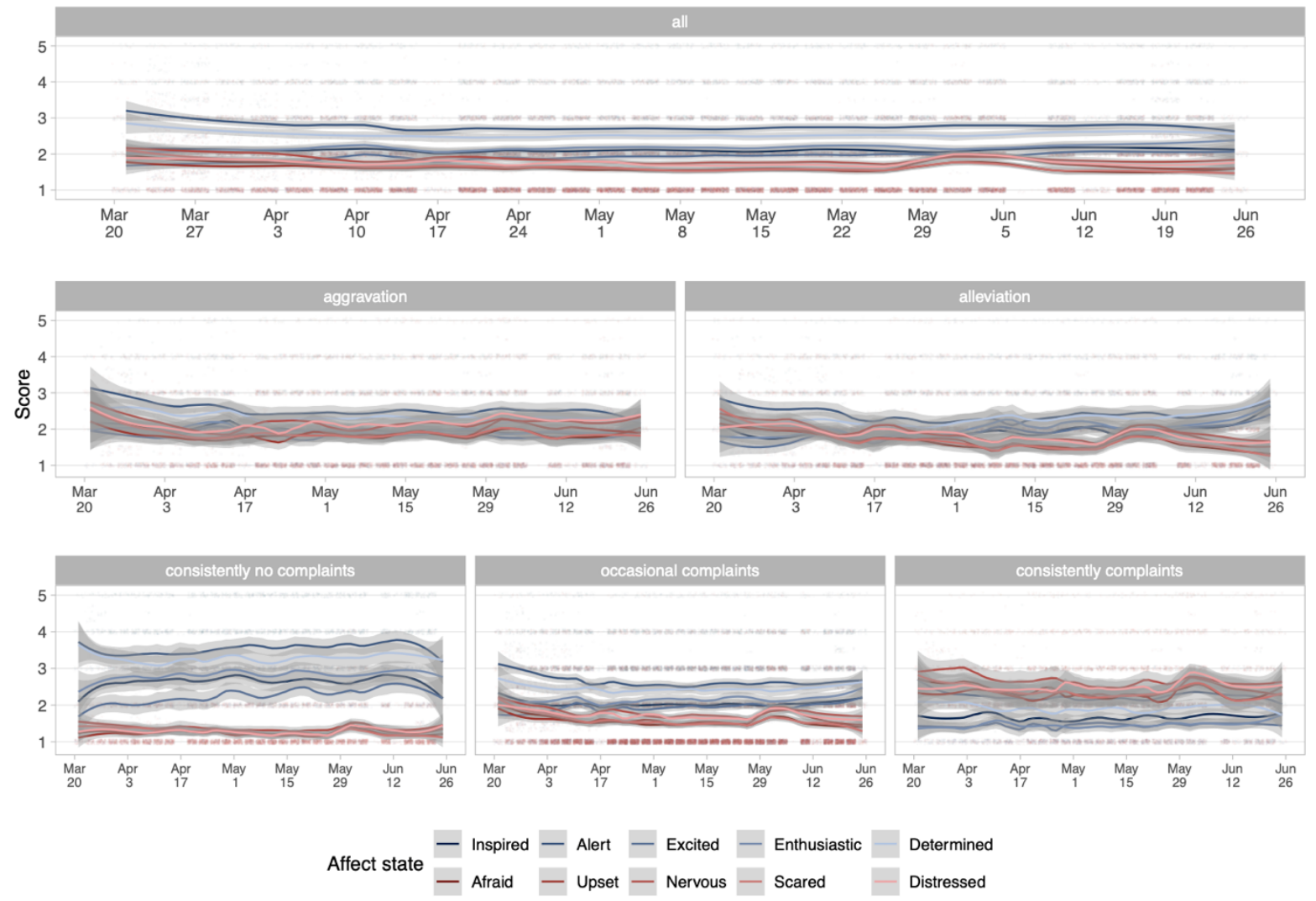

Figure 1. Affect trajectories from March 20th 2020 (measurement occasion 1) until June $26^{\text {th }} 2020$ (measurement occasion 33). Panel (a) shows the smoothed conditional mean trajectories of all participants for each of the affect states, together with its 95\% confidence interval (shaded area). Panel (b) on the left shows the trajectories for participants whose depressive complaints aggravated during the study period, and on right shows the trajectories for participants whose depressive complaints alleviated during the study period. The (c) panels show the trajectories for participants who consistently experienced no depressive complaints (left), those that occasionally experienced at least mild depressive complaints (middle), and those that consistently experienced depressive complaints (right). Blue lines correspond to the smoothed conditional means of the positive affect states 'inspired', 'alert', 'excited', 'enthusiastic', and 'determined'; and red lines correspond to the smoothed conditional means of the negative affect states 'afraid', 'upset', 'nervous', 'scared', and 'distressed'. Affect states are scored on a Likert scale from 1 to 5. Decimal scores were obtained when participants completed multiple assessments within one measurement occasion.

Third, we investigated the dynamical relations among affect states and depressive complaints by estimating a multilevel network model. Figure 2 shows the three estimated network structures: (a) the temporal associations averaged over all participants; (b) the contemporaneous associations averaged over all participants; and (c) the between-persons network structure. The temporal network shows many associations between the affect states and depressive complaints, indicating a direct interplay between affect and depression. Compared with the temporal network, the contemporaneous network shows clearer demarcations between PA, NA and depressive complaints: there are comparatively stronger edges within than between the three domains. This might indicate that within one measurement occasion the experience 
of PA, NA, and depressive complaints is relatively independent. The between-persons network portrays more relations between the different domains compared with the contemporaneous network, indicating that the average affect states people experience are related to their average depressive complaints.
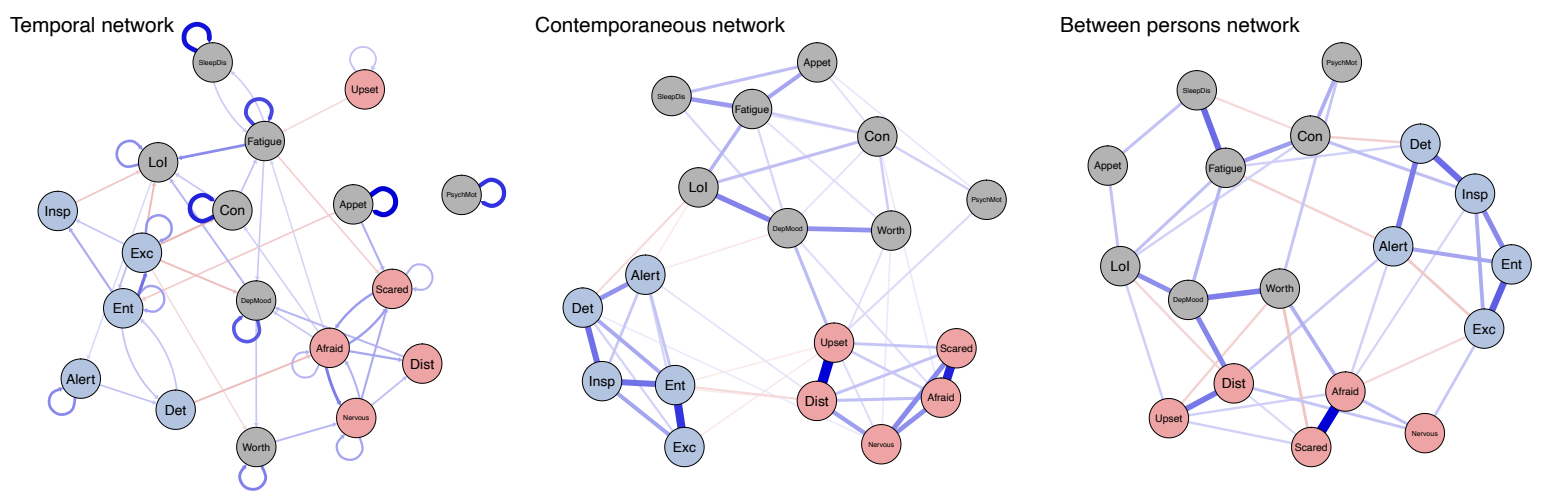

Figure 2. Output from mlVAR for $n=228$ and $t>20$. Left, the temporal network model is displayed, portraying the average within-person relations from one measurement occasion onto the next. The center displays the contemporaneous network model, portraying the average within-person effects in the same measurement occasion, after controlling for the temporal effects. Right, the between-persons network model is displayed, indicating the average effects between persons. Blue edges indicate positive relations, whereas red edges indicate negative relations. Node colors correspond to PA (light blue), NA (salmon pink), and depression complains (grey). Abbreviations: Insp $=$ inspired; Alt $=$ alert Exc $=$ excited Ent $=$ enthusiastic; Det $=$ determined $;$ Afr $=$ afraid; Ups $=$ upset; Ner = nervous; Scar $=$ scared; Dist $=$ distressed; LoI $=$ loss of interest; DepMood $=$ depressed mood; SleepDis $=$ sleep disturbances; Appet $=$ loss of appetite; Worth $=$ feelings of worthlessness; Con $=$ concentration problems; PsychMot $=$ psychomotor agitation or retardation.

Interestingly, the separation between the different network components (i.e., PA, NA, and depressive complaints) seems less evident in the temporal network compared to the contemporaneous and betweenpersons network; there are relatively more edges between components vs. within components within the temporal network. Clustering analyses confirmed the original three components in the contemporaneous network and the between-person network; however, these components were not confirmed for the temporal network (see Supplement B, Figure 3-4). Depending on the clustering algorithm (spinglass or walktrap), 4 to 5 clusters were found within the average temporal network. These clusters were a mix of different affect states and depressive complaints.

Lastly, to further investigate the relations between affect states and depressive complaints, we correlated individuals' network density of the temporal network to their maximum change in PHQ-9 score. As shown from the correlation plot in Figure 3 (right panel), there is a strong correlation $(R=0.77)$ between individual network density and maximum absolute change in PHQ-9 score. Stronger network densities relate to both a more substantial aggravation and to a more substantial alleviation in their depressive complaints, as can be seen from the bifurcation in Figure 3 (left panel) illustrating the correlation between individual network density and change in PHQ-9 score. 
PREPRINT WITHOUT PEER-REVIEW
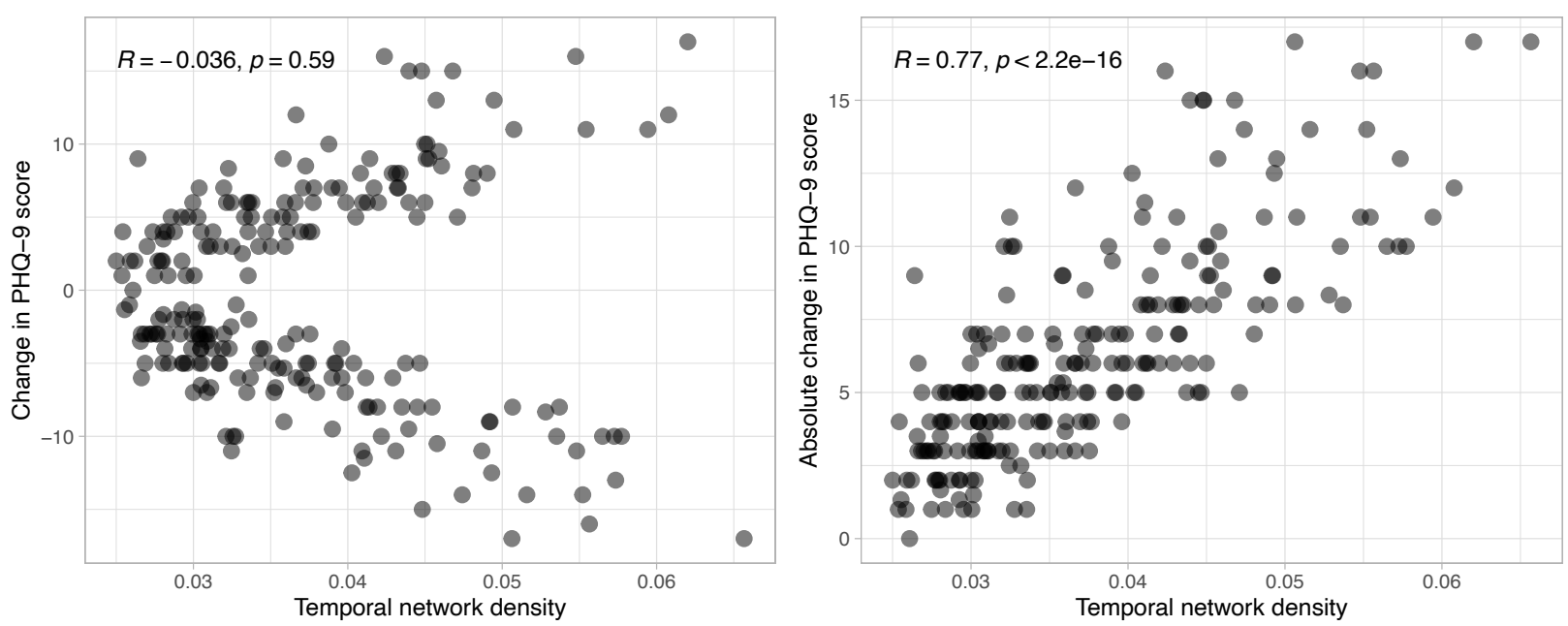

Figure 3. In the left panel the correlation between individual network density and maximum change in PHQ-9 score is shown. In the right panel the correlation between individual network density and the absolute maximum change in PHQ-9 score is shown.

\section{Discussion}

In the current paper we set-out to investigate the dynamic interplay between affect states and depressive complaints during a prolonged and eventful time imposed by the COVID-19 pandemic. The unprecedented dataset allowed us to investigate how affect fluctuated over time, for different levels of depression severity and consistency. At first sight, affect fluctuations seem relatively stable over time, in which, on average, participants reported somewhat higher levels of PA than NA. However, we found different results when separating the affect trajectories based on the participant's depressive complaints. Interestingly, while the affect trajectories were similar for people experiencing either an aggravation or alleviation of depressive complaints, we found marked differences in the trajectories among people who experienced consistently no depressive complaints compared with consistent depressive complaints. Specifically, the experienced affect seemed to 'flip' depending on the severity of one's depressive complaints. Crucially, these differences pertained to both PA and NA trajectories, showing that there is a clear link between depressive complaints and both positive and negative affect.

We subsequently investigated the link between affect and depression more directly, and network models revealed many and strong relations between the affect and depressive complaints, showing that both are indeed directly linked, both within- and across persons, and both within a single time point and over time. We identified three clear clusters of variables - pertaining to PA, NA and depressive complaints - in the contemporaneous and between-subject networks. Interestingly however, we did not find these clusters in the temporal network. This suggests that the relations among affect and depressive complaints within persons over time may be substantially different from their relations within one timepoint or betweenpersons.

Furthermore, we found a strong relation between the strength of the links in an individual's temporal network (i.e., the density) and the absolute change in PHQ-9 score. Interestingly, when considering the direction of change - alleviation or aggravation in PHQ-9 score - a bifurcation appeared, indicating that 
the same network density can relate to either a worsening or improvement in depression complaints. Crucially, this finding reflects a well-known property of test reliability, namely that the variance of a total score (in our case the change in depression complaints) consists of the sum over the variance in all items (in our case the individual affect states and individual PHQ-9 items) and the sum over their covariances (Cronbach, 1951). Clearly, denser networks indicate stronger covariances, that is necessarily reflected in the variation of the sum score (i.e., the variation in PHQ-9 score). While this is a statistical necessity, this is, to the best of our knowledge, the first time that it has been shown in relation to psychopathology networks.

Some limitations warrant attention. First, the questionnaires were sent out twice a week at random intervals, thereby violating the assumption of equidistant measures for longitudinal analyses. We circumvented this problem in part by defining measurement occasions as three-day periods. Second, it should be noted that while we are interested in mechanisms of change, the current available network estimation techniques assume that the mean and variance of the time series data remains the same (i.e., stationarity) (Jordan et al., 2020). However, alternative time-varying network models require many more datapoints than present in the current dataset (e.g., see Haslbeck et al., 2020). Therefore, there is a mismatch between our data, our interest in change, and the available statistical models.

To conclude, we found that fluctuations of affect states are directly related to the course of depressive complaints, both within- and across individuals, and both within a single measurement moment and over time. At first sight, affect trajectories over time seemed stable, even in the eventful time of COVID-19. However, marked differences in the affect trajectories appeared when separating individuals based on the consistency and severity of their depressive complaints. The conducted network analyses showed that there are direct interactions between affect states, for both PA and NA, and depression complaints. The direct links between these domains was found when averaging over all individuals, and both within one measurement moment as over time. On the individual level, we showed that the stronger that affect states and depressive complaints are overall connected over time, the larger the change is in depressive complaints. Together, these findings shed light on the potential underlying mechanisms of change and development of mental disorders. 


\section{References}

Abrams, D. B., Turner, J. R., Baumann, L. C., Karel, A., Collins, S. E., Witkiewitz, K., Fulmer, T., Tanenbaum, M. L., Commissariat, P., Kupperman, E., Baek, R. N., Gonzalez, J. S., Brandt, N., Flurie, R., Heaney, J., Kline, C., Carroll, L., Upton, J., Buchain, P. C., ... Wiebe, D. J. (2013). Affect. In Encyclopedia of Behavioral Medicine (pp. 49-50). Springer New York. https://doi.org/10.1007/978-1-4419-1005-9_1088

Bale, T. L. (2006). Stress sensitivity and the development of affective disorders. Hormones and Behavior, 50(4), 529-533. https://doi.org/10.1016/j.yhbeh.2006.06.033

Borsboom, D. (2017). A network theory of mental disorders. World Psychiatry, 16(1), 5-13. https://doi.org/10.1002/wps.20375

Borsboom, D., \& Cramer, A. O. J. (2013). Network Analysis: An Integrative Approach to the Structure of Psychopathology. Annual Review of Clinical Psychology, 9(1), 91-121. https://doi.org/10.1146/annurev-clinpsy-050212-185608

Brown, S. M., Doom, J. R., Lechuga-Peña, S., Watamura, S. E., \& Koppels, T. (2020). Stress and parenting during the global COVID-19 pandemic. Child Abuse and Neglect, 110, 104699. https://doi.org/10.1016/j.chiabu.2020.104699

Calabrese, F., Molteni, R., Racagni, G., \& Riva, M. A. (2009). Neuronal plasticity: A link between stress and mood disorders. In Psychoneuroendocrinology (Vol. 34, Issue SUPPL. 1, pp. S208-S216). Pergamon. https://doi.org/10.1016/j.psyneuen.2009.05.014

Cleveland, W. S. (1979). Robust locally weighted regression and smoothing scatterplots. Journal of the American Statistical Association, 74(368), 829-836. https://doi.org/10.1080/01621459.1979.10481038

Cramer, A. O. J., van Borkulo, C. D., Giltay, E. J., van der Maas, H. L. J., Kendler, K. S., Scheffer, M., \& Borsboom, D. (2016). Major Depression as a Complex Dynamic System. PLOS ONE, 11(12), e0167490. https://doi.org/10.1371/journal.pone.0167490

Cronbach, L. J. (1951). Coefficient alpha and the internal structure of tests. Psychometrika, 16, 297-334.

Cunningham, T. J., Fields, E. C., \& Kensinger, E. A. (2021). Boston College daily sleep and well-being survey data during early phase of the COVID-19 pandemic. Scientific Data, 8(1), 1-6. https://doi.org/10.1038/s41597-021-00886-y

Dejonckheere, E., Kalokerinos, E. K., Bastian, B., \& Kuppens, P. (2019). Poor emotion regulation ability mediates the link between depressive symptoms and affective bipolarity. Cognition and Emotion, 33(5), 1076-1083. https://doi.org/10.1080/02699931.2018.1524747

Ebrahimi, O. V., Burger, J., Hoffart, A., \& Johnson, S. U. (n.d.). Within and across-day patterns of interplay between depressive symptoms and related psychopathological processes: A dynamic network approach during the COVID-19 pandemic. https://doi.org/10.31234/OSF.IO/AF5DH

Epskamp, S., Borsboom, D., \& Fried, E. I. (2018). Estimating psychological networks and their accuracy: A tutorial paper. Behavior Research Methods, 50(1), 195-212. https://doi.org/10.3758/s13428-0170862-1

Epskamp, S., Deserno, M. K., \& Bringmann, L. F. (2017). mlVAR: multi-level vector autoregression. $R$ Package Version 0.3.

Epskamp, S., van Borkulo, C. D., van der Veen, D. C., Servaas, M. N., Isvoranu, A. M., Riese, H., \& Cramer, A. O. J. (2018a). Personalized Network Modeling in Psychopathology: The Importance of Contemporaneous and Temporal Connections. Clinical Psychological Science, 6(3), 416-427. https://doi.org/10.1177/2167702617744325

Epskamp, S., van Borkulo, C. D., van der Veen, D. C., Servaas, M. N., Isvoranu, A. M., Riese, H., \& Cramer, A. O. J. (2018b). Personalized Network Modeling in Psychopathology: The Importance of Contemporaneous and Temporal Connections. Clinical Psychological Science, 6(3), 416-427. https://doi.org/10.1177/2167702617744325

Epskamp, S., Waldorp, L. J., Mõttus, R., \& Borsboom, D. (2018). The Gaussian Graphical Model in Cross-Sectional and Time-Series Data. Multivariate Behavioral Research, 53(4), 453-480. 
PREPRINT WITHOUT PEER-REVIEW

https://doi.org/10.1080/00273171.2018.1454823

Fredrickson, B. L. (2004). The broaden-and-build theory of positive emotions. Philosophical Transactions of the Royal Society B: Biological Sciences, 359(1449), 1367-1377. https://doi.org/10.1098/rstb.2004.1512

Garnefski, N., \& Kraaij, V. (2006). Relationships between cognitive emotion regulation strategies and depressive symptoms: A comparative study of five specific samples. Personality and Individual Differences, 40(8), 1659-1669. https://doi.org/10.1016/j.paid.2005.12.009

Grolli, R. E., Mingoti, M. E. D., Bertollo, A. G., Luzardo, A. R., Quevedo, J., Réus, G. Z., \& Ignácio, Z. M. (2021). Impact of COVID-19 in the Mental Health in Elderly: Psychological and Biological Updates. In Molecular Neurobiology (Vol. 58, Issue 5, pp. 1905-1916). Springer. https://doi.org/10.1007/s12035-020-02249-x

Gross, J. J. (1999). Emotion regulation: Past, present, future. Cognition and Emotion, 13(5), 551-573. https://doi.org/10.1080/026999399379186

Guille, C., Clark, S., Amstadter, A. B., \& Sen, S. (2014). Trajectories of depressive symptoms in response to prolonged stress in medical interns. Acta Psychiatrica Scandinavica, 129(2), 109-115. https://doi.org/10.1111/acps.12137

Han, X., Chen, S., Bi, K., Yang, Z., \& Sun, P. (2021). Depression Following COVID-19 Lockdown in Severely, Moderately, and Mildly Impacted Areas in China. Frontiers in Psychiatry, 12, 596872. https://doi.org/10.3389/fpsyt.2021.596872

Haslbeck, J. M. B., Bringmann, L. F., \& Waldorp, L. J. (2020). A Tutorial on Estimating Time-Varying Vector Autoregressive Models. Https://Doi.Org/10.1080/00273171.2020.1743630, 56(1), 120-149. https://doi.org/10.1080/00273171.2020.1743630

Hölzel, L., Härter, M., Reese, C., \& Kriston, L. (2011). Risk factors for chronic depression - A systematic review. In Journal of Affective Disorders (Vol. 129, Issues 1-3, pp. 1-13). Elsevier. https://doi.org/10.1016/j.jad.2010.03.025

Joormann, J., \& Gotlib, I. H. (2010). Emotion regulation in depression: Relation to cognitive inhibition. Cognition \& Emotion, 24(2), 281-298. https://doi.org/10.1080/02699930903407948

Joormann, J., \& Stanton, C. H. (2016). Examining emotion regulation in depression: A review and future directions. In Behaviour Research and Therapy (Vol. 86, pp. 35-49). Elsevier Ltd. https://doi.org/10.1016/j.brat.2016.07.007

Jordan, D. G., Winer, E. S., \& Salem, T. (2020). The current status of temporal network analysis for clinical science: Considerations as the paradigm shifts? Journal of Clinical Psychology, jclp.22957. https://doi.org/10.1002/jclp.22957

Kalisch, R., Baker, D. G., Basten, U., Boks, M. P., Bonanno, G. A., Brummelman, E., Chmitorz, A., Fernàndez, G., Fiebach, C. J., Galatzer-Levy, I., Geuze, E., Groppa, S., Helmreich, I., Hendler, T., Hermans, E. J., Jovanovic, T., Kubiak, T., Lieb, K., Lutz, B., ... Kleim, B. (2017). The resilience framework as a strategy to combat stress-related disorders. In Nature Human Behaviour (Vol. 1, Issue 11, pp. 784-790). Nature Publishing Group. https://doi.org/10.1038/s41562-017-0200-8

Kaufman, K. R., Petkova, E., Bhui, K. S., \& Schulze, T. G. (2020). A global needs assessment in times of a global crisis: world psychiatry response to the COVID-19 pandemic. BJPsych Open, 6(3). https://doi.org/10.1192/bjo.2020.25

Khazanov, G. K., \& Ruscio, A. M. (2016). Is low positive emotionality a specific risk factor for depression? A meta-Analysis of longitudinal studies. Psychological Bulletin, 142(9), 991-1015. https://doi.org/10.1037/bul0000059

Kocevska, D., Blanken, T. F., Van Someren, E. J. W., \& Rösler, L. (2020). Sleep quality during the COVID-19 pandemic: not one size fits all. Sleep Medicine, 76, 86-88. https://doi.org/10.1016/j.sleep.2020.09.029

Kramer, I., Simons, C. J. P., Wigman, J. T. W., Collip, D., Jacobs, N., Derom, C., Thiery, E., van Os, J., Myin-Germeys, I., \& Wichers, M. (2014). Time-Lagged Moment-to-Moment Interplay Between Negative Affect and Paranoia: New Insights in the Affective Pathway to Psychosis. Schizophrenia Bulletin, 40(2), 278-286. https://doi.org/10.1093/SCHBUL/SBS194 
Kroenke, K., Spitzer, R. L., \& Williams, J. B. W. (2001). The PHQ-9: Validity of a brief depression severity measure. Journal of General Internal Medicine, 16(9), 606-613. https://doi.org/10.1046/j.1525-1497.2001.016009606.x

Kuranova, A., Booij, S. H., Menne-Lothmann, C., Decoster, J., van Winkel, R., Delespaul, P., De Hert, M., Derom, C., Thiery, E., Rutten, B. P. F., Jacobs, N., van Os, J., Wigman, J. T. W., \& Wichers, M. (2020). Measuring resilience prospectively as the speed of affect recovery in daily life: a complex systems perspective on mental health. BMC Medicine 2020 18:1, 18(1), 1-11. https://doi.org/10.1186/S12916-020-1500-9

Lowe, B., Unutzer, J., Callahan, C., Perkins, A., \& Kroenke, K. (2004). Monitoring depression treatment outcomes with the patient health questionnaire-9. Medical Care, 42(12), 194-201.

Mackinnon, A., Jorm, A. F., Christensen, H., Korten, A. E., Jacomb, P. A., \& Rodgers, B. (1999). A short form of the Positive and Negative Affect Schedule: evaluation of factorial validity and invariance across demographic variables in a community sample. Personality and Individual Differences, 27(3), 405-416. https://doi.org/10.1016/S0191-8869(98)00251-7

McEwen, B. S. (2000). Allostasis and allostatic load: Implications for neuropsychopharmacology. Neuropsychopharmacology, 22(2), 108-124. https://doi.org/10.1016/S0893-133X(99)00129-3

Moore, C., \& Kolencik, J. (2020). Acute Depression, Extreme Anxiety, and Prolonged Stress among COVID-19 Frontline Healthcare Workers. Psychosociological Issues in Human Resource Management, 8(1), 55. https://doi.org/10.22381/pihrm8120209

Oreel, T. H., Borsboom, D., Epskamp, S., Hartog, I. D., Netjes, J. E., Nieuwkerk, P. T., Henriques, J. P. S., Scherer-Rath, M., van Laarhoven, H. W. M., \& Sprangers, M. A. G. (2019). The dynamics in health-related quality of life of patients with stable coronary artery disease were revealed: a network analysis. Journal of Clinical Epidemiology, 107, 116-123. https://doi.org/10.1016/J.JCLINEPI.2018.11.022

Pfefferbaum, B., \& North, C. S. (2020). Mental Health and the Covid-19 Pandemic. New England Journal of Medicine, 383(6), 510-512. https://doi.org/10.1056/nejmp2008017

Round, J. M., Lee, C., Hanlon, J. G., Hyshka, E., Dyck, J. R. B., \& Eurich, D. T. (2020). Changes in patient health questionnaire (PHQ-9) scores in adults with medical authorization for cannabis. BMC Public Health, 20(987). https://doi.org/10.1186/s12889-020-09089-3

Schoevers, R. A., Van Borkulo, C. D., Lamers, F., Servaas, M. N., Bastiaansen, J. A., Beekman, A. T. F., Van Hemert, A. M., Smit, J. H., Penninx, B. W. J. H., \& Riese, H. (2020). Affect fluctuations examined with ecological momentary assessment in patients with current or remitted depression and anxiety disorders. Psychological Medicine, 1-10. https://doi.org/10.1017/S0033291720000689

Van Lancker, W., \& Parolin, Z. (2020). COVID-19, school closures, and child poverty: a social crisis in the making. In The Lancet Public Health (Vol. 5, Issue 5, pp. e243-e244). Elsevier Ltd. https://doi.org/10.1016/S2468-2667(20)30084-0

Watson, D., Clark, L. A., \& Tellegen, A. (1988). Development and Validation of Brief Measures of Positive and Negative Affect: The PANAS Scales. In Journal of Personality and Social Psychology (Vol. 54, Issue 6).

Wichers, M., Myin-Germeys, I., Jacobs, N., Peeters, F., Kenis, G., Derom, C., Vlietinck, R., Delespaul, P., \& Van Os, J. (2007). Genetic risk of depression and stress-induced negative affect in daily life. British Journal of Psychiatry, 191(3), 218-223. https://doi.org/10.1192/bjp.bp.106.032201

Wichers, M., Peeters, F., Geschwind, N., Jacobs, N., Simons, C. J. P., Derom, C., Thiery, E., Delespaul, P. H., \& van Os, J. (2010). Unveiling patterns of affective responses in daily life may improve outcome prediction in depression: A momentary assessment study. Journal of Affective Disorders, 124(1-2), 191-195. https://doi.org/10.1016/j.jad.2009.11.010

Wichers, M., Wigman, J. T. W., \& Myin-Germeys, I. (2015). Micro-Level Affect Dynamics in Psychopathology Viewed From Complex Dynamical System Theory:

Http://Dx.Doi.Org/10.1177/1754073915590623, 7(4), 362-367.

https://doi.org/10.1177/1754073915590623 